\title{
Mortalidad atribuible a infecciones nosocomiales en un hospital de la Seguridad Social en Chiclayo, Perú
}

\section{Nosocomial infections attributable mortality in a social security hospital in Peru.}

Correspondencia Dr. Moisés Apolaya Segura moises.apolaya@gmail.com

Recibido: $12 / 09 / 2016$ Aprobado: 21/0g/2016

Citar como: Díaz-Vélez C, Neciosup-Puicán E, FernándezMogollón JL, Tresierra-Ayala MA, Apolaya-Segura M. Mortalidad atribuible a infecciones nosocomiales en un hospital de la Seguridad Social en Chiclayo, Perú. Acta Med Peru. 2016;33(3):250-2

\author{
Cristian Díaz-Vélez ${ }^{1,2}$, Elizabeth Neciosup-Puicán 1,a,Jorge Luis Fernández- \\ Mogollón ${ }^{1,2}$, Miguel Ángel Tresierra-Ayala ${ }^{3}$, Moisés Apolaya-Segura ${ }^{4}$ \\ 1 Oficina de Inteligencia Sanitaria, Hospital Nacional Almanzor Aguinaga Asenjo, EsSalud. Chiclayo, Perú. \\ 2 Facultad de Medicina, Universidad de San Martín de Porres. Chiclayo, Perú. \\ 3 Hospital Víctor Lazarte Echegaray. Trujillo, Perú. \\ 4 Facultad de Medicina, Universidad de San Martín de Porres. Lima, Perú. \\ a Enfermera
}

\section{Sr. Editor:}

Las infecciones nosocomiales o intrahospitalarias (IIH) actualmente llamadas infecciones asociadas a la atención en salud (IAAS) son causa importante de morbilidad, aunque se desconoce la carga de enfermedad producida en la región de las Américas ${ }^{[1]}$ l la mortalidad supera el $50 \%$ a nivel mundial ${ }^{[2]}$. LasIAASson aquellas infecciones que el paciente adquiere mientras recibe tratamiento para alguna condición médica o quirúrgica y en quien la infección no se había manifestado ni estaba en periodo de incubación en el momento del ingreso a la institución, con la presencia de signos o síntomas sugestivos de infección a partir del tercer día de hospitalización ${ }^{[3]}$. Una muerte hospitalaria atribuible a infección nosocomial, cuando en el proceso de muerte se involucra esta infección ${ }^{[4]}$.

En el Perú, un estudio de la Dirección General de Epidemiología del Ministerio de Salud, reporta tasas de prevalencia de IIH de 4,8\% para el 2014 Y 3,9\% para el 2015, y tasa de prevalencia de pacientes con IIH de 4,4Y 3,6\% para los mismos años(www. minsa.gob.pe/dgsp/). En Chiclayo-Perú el Hospital Nacional Almanzor Aguinaga Asenjo (HNAAA) es un hospital de la Seguridad Social que recibe referencias de toda la región norte del país y tiene reportado en el 2010 una tasa de prevalencia de infecciones intrahospitalarias de $9,4 \%$ con una tendencia estacionaria entre los años 2011-2015 $5^{[5]}$.

La tasa bruta de mortalidad hospitalaria en la Seguridad Social a nivel nacional llega a 2,3\%, que fluctúa de hospitales nivel I $(0,7 \%)$ hasta $4,2 \%$ en hospitales nacionales (www.essalud.gob.pe).Existen varios estudios que muestran que la mortalidad atribuible a infección nosocomial varía entre $14,6 \%{ }^{[6]} y$ $21,3 \% 0^{[4]}$. En el HNAAA (Figura 1) presentó una tendencia en aumento de esta tasa, 15,8\% el 2013, 17,5\% el 2014 y 18,5\% en el año 2015; sinembargo, en el primer semestre del 2016 se nota una disminución significativa a $13,3 \%(p<0,01)$.

La Seguridad Social reportó 23909 muertes hospitalarias durante el 2015 en los centros asistenciales a nivel nacional(www.essalud.gob.pe), si tomamos el promedio $17,3 \%$ de nuestro periodo del 2013-2015aproximadamente 4137 muertes se habrían presentado a nivel nacional atribuidas a infección nosocomial.

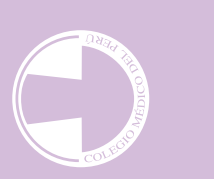


Tabla 1. Factores asociados a infecciones nosocomiales involucradas en mortalidad hospitalaria, Hospital Nacional Almanzor Aguinaga Asenjo 2013-2016.

\begin{tabular}{|c|c|c|c|c|c|c|c|}
\hline \multirow{3}{*}{ Factores } & \multicolumn{4}{|c|}{ Muerte Intrahospitalaria } & \multirow{3}{*}{ RP } & \multirow{3}{*}{$(\mathrm{IC} 95 \%)$} & \multirow{3}{*}{ p } \\
\hline & \multicolumn{2}{|c|}{ Asociada a IIH n=743 } & \multicolumn{2}{|c|}{$\begin{array}{c}\text { No asociado a IIH } \\
n=3710\end{array}$} & & & \\
\hline & $\mathbf{n}$ & $(\%)$ & $\mathbf{n}$ & $(\%)$ & & & \\
\hline \multicolumn{8}{|l|}{ Edad } \\
\hline 0-8 años & 17 & $(3,9)$ & 416 & $(96,1)$ & 0,19 & $(0,09-0,41)$ & $p<0,05$ \\
\hline 9-17 años & 9 & $(20,0)$ & 36 & $(80,0)$ & 1,00 & Referencia & \\
\hline 18-59 años & 113 & $(13,4)$ & 733 & $(86,6)$ & 0,66 & $(0,36-1,22)$ & $p>0,05$ \\
\hline$>60$ años & 604 & $(19,3)$ & 2525 & $(80,7)$ & 0,96 & $(0,53-1,73)$ & $p>0,05$ \\
\hline \multicolumn{8}{|l|}{ Género } \\
\hline Masculino & 422 & $(17,5)$ & 1983 & $(82,5)$ & 0,92 & $(0,84-1,01)$ & $p>0,05$ \\
\hline Femenino & 321 & $(15,7)$ & 1727 & $(84,3)$ & 1,00 & Referencia & \\
\hline \multicolumn{8}{|l|}{ Servicio crítico } \\
\hline Sí & 395 & $(15,0)$ & 2236 & $(85,0)$ & 1,27 & $(1,11-1,45)$ & $p<0,05$ \\
\hline No & 348 & $(19,1)$ & 1474 & $(80,9)$ & 1,00 & Referencia & \\
\hline \multicolumn{8}{|l|}{ Servicio } \\
\hline Quirúrgico & 69 & $(31,9)$ & 147 & $(68,1)$ & 9,1 & $(6,7-12,5)$ & $p<0,05$ \\
\hline Clínico & 613 & $(24,7)$ & 1870 & $(75,3)$ & 7,09 & $(5,49-9,1)$ & $p<0,05$ \\
\hline Clínico- Quirúrgico & 61 & $(83,5)$ & 1693 & $(96,5)$ & 1,00 & Referencia & \\
\hline \multicolumn{8}{|l|}{ Motivo de hospitalización } \\
\hline Causas externase & 48 & $(26,7)$ & 132 & $(73,3)$ & 3,00 & $(1,62-5,55)$ & $p<0,05$ \\
\hline Sistema nervioso & 20 & $(23,8)$ & 64 & $(76,2)$ & 2,68 & $(1,35-5,30)$ & $p<0,05$ \\
\hline Diabetes mellitus & 55 & $(22,4)$ & 191 & $(77,6)$ & 2,52 & $(1,36-4,63)$ & $p<0,05$ \\
\hline Sistema genitourinario & 46 & $(21,6)$ & 167 & $(78,4)$ & 2,43 & $(1,31-4,52)$ & $p<0,05$ \\
\hline Cáncer & 208 & $(19,2)$ & 876 & $(80,8)$ & 2,16 & $(1,21-3,85)$ & $p<0,05$ \\
\hline Sistema digestivo & 100 & $(20,2)$ & 394 & $(79,8)$ & 2,28 & $(1,26-4,11)$ & $p<0,05$ \\
\hline Afecciones perinatales & 3 & $(1,2)$ & 237 & $(98,8)$ & 0,14 & $(0,04-0,49)$ & $p<0,05$ \\
\hline Sistema circulatorio & 147 & $(16,3)$ & 756 & $(83,7)$ & 1,83 & $(1,02-3,28)$ & $p>0,05$ \\
\hline Embarazo, parto y puerperio & 1 & $(14,3)$ & 6 & $(85,7)$ & 1,61 & $(0,24-10,76)$ & $p>0,05$ \\
\hline Malformaciones congénitas & 4 & $(3,0)$ & 128 & $(97,0)$ & 0,34 & $(0,11-1,04)$ & $p>0,05$ \\
\hline Infecciosa & 11 & $(8,9)$ & 113 & $(91,1)$ & 1,00 & Referencia & \\
\hline
\end{tabular}

${ }^{E}$ Causas externas: (accidentes, agresiones, etc)

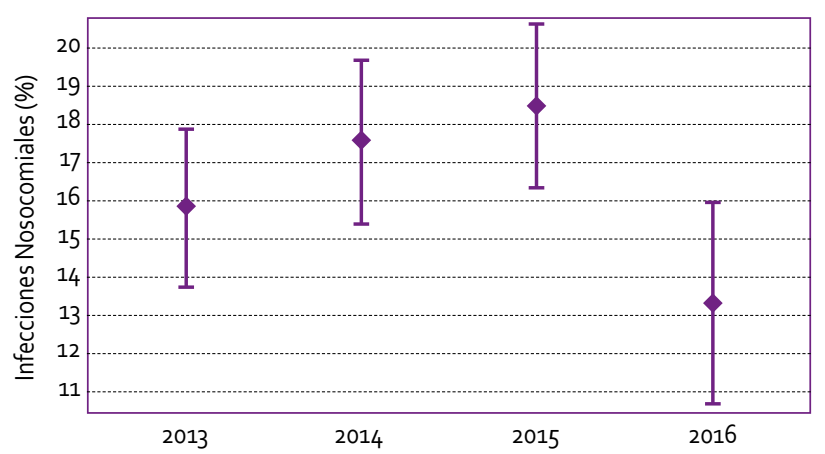

Figura1. Frecuencia de las infecciones nosocomiales asociadas a mortalidad hospitalaria, Hospital Nacional Almanzor Aguinaga Asenjo 2013-2016

Fuente: Subsistema de Vigilancia de Mortalidad Hospitalaria, Hospital Nacional Almanzor Aguinaga Asenjo
Son varios los factores asociados a infecciones intrahospitalaria, reportados en la literatura científica ${ }^{[7]}$, recibir tratamiento antibiótico inadecuado aporta mayor riesgo de mortalidad, internamiento en un servicio hospitalario critico dado que ellos sufren de enfermedad severa y aguda antes del inicio de la infección, sin embargo aun así también resulta devastador; con alta mortalidad, pero quepuede ser controlada por la implementación de medidas generales de prevención y una política de uso de antimicrobianos en forma adecuada como lo recomiendan las guías basada en evidencia de bioseguridad.

Durante los años 2014-2015 varios estudios operativos que mostraban el incumplimiento de las medidas básicas de prevención como no lavado de manos en forma adecuada ${ }^{[8]}$, inadecuada limpieza hospitalaria ${ }^{[9]}$ y presencia de gérmenes con resistencia antimicrobiana BLEE positivo en casi $63 \%$ de las muestras y aun así la ceftriaxona era el antibiótico más 
usado ${ }^{[10]}$, así como evaluar el sistema de vigilancia; acciones que probablemente llevaron al aumento sostenido de la mortalidad hospitalaria atribuido a infecciones nosocomiales entre el 2013 al 2015, por lo que se realizó un análisis de la información registrada en el Subsistema de Vigilancia de Mortalidad Hospitalaria para encontrar los factores asociados al involucramiento de las infecciones intrahospitalarias en la mortalidad, además de las ya encontradas en los estudios operativos, definiendo a causa atribuible si luego del análisis del expediente clínico realizado se registraba como causa intermedia o final de defunción (Tabla 1)

Entre los hallazgos se tienen que la edad entre o-8 años disminuye $81 \%$ la probabilidad de $\mathrm{IHH}$ involucrada en la mortalidad hospitalaria, la hospitalización en un servicio crítico aumenta $27 \%$ esa probabilidad. Algunos enfermedades de fondo aumentan la probabilidad de tener una infección nosocomial involucrada en su proceso de muerte, cáncer (RP: 2,16; IC95\%:1,21-3,85), accidentes y agresiones (RP: 3, IC95\%:1.62-5.55), diabetes mellitus (RP: 2,52; IC95\%:1,36-4,63), afecciones del sistema digestivo (RP: 2,28; IC95\%:1, 26-4,11), genitourinario (RP: 2,43; IC $95 \%: 1,31-4,52)$ y sistema nervioso (RP: 2,$68 ;$ IC $95 \%: 1,35-5,30$ ).

Es importante mencionar que durante el segundo semestre del año 2015 y primer semestre del 2016 se realizaron actividades educacionales y motivacionales en lavado de manos, talleres de infecciones intrahospitalarias, rondas de seguridad del paciente, intervenciones oportunas en brotes de infecciones intrahospitalarias, abastecimiento de insumos en bioseguridad, entre otras actividades que probablemente hayan tenido un impacto en la reducción de la mortalidad atribuible a infecciones nosocomiales.

Culminar resaltando la vigencia y la importancia de las medidas de bioseguridad en un medio donde contamos con pocos recursos económicos y con el alto impacto económico de las infecciones asociadas a la atención en salud y la vigilancia de su adherencia llegan a tener un buen impacto en la morbilidad por esas infecciones y su involucramiento en la mortalidad hospitalaria.
Fuente de financiamiento: Los autores declaran no haber recibido ninguna financiación para la realización de este trabajo.

Conflicto de intereses: Los autores declaran no tener conflicto de intereses con la publicación de este artículo.

\section{REFERENCIAS BIBLIOGRÁFICAS}

1. Organización Panamericana de la Salud. Vigilancia epidemiológica de las infecciones asociadas a la atención de la salud. Módulo III: información para gerentes y personal directivo. Washington DC: OPS; 2012

2. Guimarães $A C$, Donalisio $M R$, Santiago $T H R$, Freire JB. [Mortality associated with nosocomial infection, occurring in a general hospital of Sumaré-SP, Brazil]. Rev Bras Enferm. 2011;64(5):864-9.

3. Kouchak F, Askarian M. Nosocomial infections: the definition criteria. Iran J Med Sci. 2012;37(2):72-3.

4. García-Martín $M$, Lardelli-Claret $P$, Jiménez-Moleón JJ, BuenoCavanillas A, Luna-del-Castillo JD, Gálvez-Vargas R. Proportion of hospital deaths potentially attributable to nosocomial infection. Infect Control Hosp Epidemiol. 2001;22(11):708-14.

5. Díaz-Vélez C. Las infecciones nosocomiales, un problema vigente. Rev Cuerpo Med HNAAA. 2016;9(1):4-5.

6. Kaoutar B, Joly C, L'Hériteau F, Barbut F, Robert J, Denis M, et al. Nosocomial infections and hospital mortality: a multicentre epidemiology study. J Hosp Infect. 2004; 58(4):268-75.

7. Sheng W-H, Wang J-T, Lin M-S, Chang S-C. Risk factors affecting in-hospital mortality in patients with nosocomial infections. J Formos Med Assoc. 2007;106(2):110-8.

8. Polo-Capuñay AM, Soto-Caceres V, Díaz-Vélez C. Cumplimiento de "care bundle" para prevención de infección del torrente sanguíneo asociado a catéter intravascular en el HNAAA, enero-febrero, 2015. Rev Cuerpo Med HNAAA. 2015;8(3):162-7.

9. Failoc-Rojas VE, Molina-Ayasta C, Díaz-Velez C. Importancia de la limpieza hospitalaria para el control de infecciones intrahospitalarias: evaluación microbiológica de un hospital de Chiclayo, Perú. Infectio. 2015;19(4):183-184.

10. Fernández-Mogollón JL, Tello-Vera S, Pizarro-Chima F. Perfil Microbiológico de un Hospital del Seguro Social Nivel III, ChiclayoPerú. 2014. Rev Cuerpo Med HNAAA. 2016;9(1):6-13.

\section{Ahora puede enviar sus artículos para Acta Médica Peruana en nuestro Open Journal System:}

\section{www.amp.cmp.org.pe}

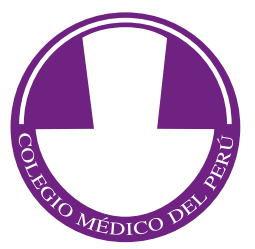

